\title{
In Vivo $\mathrm{T}_{2}$ Relaxation Time Measurement with Echo-Time Averaging
}

\author{
Andrew P. Prescot ${ }^{1,2}$, Xianfeng Shi ${ }^{1,3}$, Changho Choi $^{4}$, and Perry. F. Renshaw ${ }^{1,3,5}$ \\ Andrew P. Prescot: andrew.prescot@utah.edu; Xianfeng Shi: xianfeng.shi@hsc.utah.edu; Changho Choi: \\ Changho.Choi@utsouthwestern.edu; Perry.F.Renshaw:PERRY.RENSHAW@hsc.utah.edu \\ ${ }^{1}$ Brain Institute, University of Utah, Salt Lake City, UT, USA \\ ${ }^{2}$ Department of Radiology, University of Utah School of Medicine, Salt lake City, UT, USA \\ ${ }^{3}$ Department of Psychiatry, University of Utah School of Medicine, Salt lake City, UT, USA \\ ${ }^{4}$ Advanced Imaging Research Center, University of Texas Southwestern Medical Center, Dallas, \\ TX, USA \\ ${ }^{5}$ VISN 19 MIRECC, Salt Lake City, UT, USA
}

\begin{abstract}
The accuracy of metabolite concentrations measured using in vivo proton $\left({ }^{1} \mathrm{H}\right)$ magnetic resonance spectroscopy (MRS) is enhanced following correction for spin-spin $\left(\mathrm{T}_{2}\right)$ relaxation effects. In addition, metabolite proton $\mathrm{T}_{2}$ relaxation times provide unique information regarding cellular environment and molecular mobility. Echo-time (TE) averaging ${ }^{1} \mathrm{H}$ MRS involves the collection and averaging of multiple TE steps that greatly simplifies resulting spectra due to the attenuation of spin-coupled and macromolecule resonances. Given the simplified spectral appearance and inherent metabolite $\mathrm{T}_{2}$ relaxation information, the aim of the present proof-ofconcept study was to develop a novel data processing scheme to estimate metabolite $\mathrm{T}_{2}$ relaxation times from TE-averaged ${ }^{1} \mathrm{H}$ MRS data. Spectral simulations are used to validate the proposed TEaveraging methods for estimating methyl proton $\mathrm{T}_{2}$ relaxation times for $\mathrm{N}$-acetyl aspartate, total creatine, and choline-containing compounds. The utility of the technique and its reproducibility are demonstrated using data obtained in vivo from the posterior-occipital cortex of ten healthy control subjects. Compared to standard methods, distinct advantages of this approach include built-in macromolecule resonance attenuation, in vivo $\mathrm{T}_{2}$ estimates closer to reported values when maximum $T E \approx T_{2}$, and the potential for $T_{2}$ calculation of metabolite resonances otherwise inseparable in standard ${ }^{1} \mathrm{H}$ MRS spectra recorded in vivo.
\end{abstract}

\section{Keywords}

Proton magnetic resonance spectroscopy; Echo time averaging; Spin-spin $\left(\mathrm{T}_{2}\right)$ relaxation time

Corresponding author: Andrew P. Prescot, Brain Institute, 383 Colorow Drive, room 352, Salt Lake City, UT, 84108, USA, andrew.prescot@utah.edu tel: 801-587-1441 fax: 801-585-0575. 


\subsection{Introduction}

Proton $\left({ }^{1} \mathrm{H}\right)$ magnetic resonance spectroscopy (MRS) provides a non-invasive means for assessing metabolite concentration for a range of biologically important cerebral compounds in vivo. To extract quantitative information, metabolite MRS signal amplitudes ideally should be corrected for a number of factors related to MR-related phenomena and experimental set-up and procedures. Briefly, these factors include nuclear spin relaxation, molecular diffusion, nuclear Overhauser effects (nOe), magnetization transfer effects, radiofrequency (RF) pulse or ' $\mathrm{B}_{1}$ ' inhomogeneity, $\mathrm{RF}$ coil loading effects, and localization profile imperfections. Under certain circumstances, correction for nuclear spin relaxation is perhaps the most important step for realizing truly quantitative MRS studies. Differential signal attenuation effects due to differences in metabolite proton longitudinal or spin-lattice $\left(\mathrm{T}_{1}\right)$ relaxation times can be circumvented using sufficiently long repetition times that allow for full signal recovery between signal excitation and data collection. However, the common ${ }^{1} \mathrm{H}$ MRS pulse sequences that localize metabolite signal in three dimensions require several finite bandwidth radiofrequency pulses and incorporate one or more spin echo timing periods. The metabolite proton transverse or spin-spin $\left(T_{2}\right)$ relaxation times thus remain problematic and fitted signal amplitudes require post-hoc $\mathrm{T}_{2}$ correction. Evidenced by MRS studies in a range of pathologies, the measurement of metabolite $\mathrm{T}_{2}$ also provides complementary information regarding cellular environment and molecular motion in healthy or diseased tissue. Intracranial tumor MRS studies have shown that the trimethyl proton $\mathrm{T}_{2}$ arising from choline-containing (Cho; predominantly glycerophosphocholine and phosphorylcholine) compounds is prolonged compared to the same parameter measured in healthy tissue, indicating differences in cell membrane environment and/or metabolism (1). Ongur and colleagues reported shorter proton $\mathrm{T}_{2}$ values for anterior cingulate cortical total creatine (creatine+phosphocreatine; $\mathrm{Cre}$ ) and Cho in bipolar disorder and schizophrenia, a finding that potentially is consistent with reduced cell volume and macromolecular structures (2). These findings are further reinforced by similar studies demonstrating shortening of white matter $\mathrm{N}$-acetyl aspartate (NAA) proton $\mathrm{T}_{2}(3,4)$ as well as water proton $\mathrm{T}_{2}$ (3) in schizophrenic patients. Studies utilizing MRS for evaluating focal ischemia have revealed a modified shortening of NAA methyl proton $\mathrm{T}_{2}$ relaxation time in the weeks following infarction, which normalizes several months following stroke (5).

Localized spectroscopic $\mathrm{T}_{2}$ measurements typically involve the collection of an array of ${ }^{1} \mathrm{H}$ MRS spectra recorded with a suitable range of stepped echo time (TE) values. The metabolite signal amplitude (absolute or semilogarithmic) versus echo time data is constructed with subsequent curve fitting by a monoexponential decay function. The same data acquisition scheme is shared by two-dimensional (2D) $J$-resolved ${ }^{1} \mathrm{H}$ MRS although post-processing involves a 2D fast Fourier transformation (FFT) to afford a 2D spectral matrix characterized by metabolite $J$-coupling information versus chemical shift. Recent reports demonstrating improved acquisition techniques (i.e., maximum echo sampling, data oversampling) and automated 2D spectral fitting have significantly advanced the utility of in vivo $2 \mathrm{D}{ }^{1} \mathrm{H}$ MRS $(6,7)$. An alternative processing strategy that can be applied to $2 \mathrm{D}{ }^{1} \mathrm{H}$ MRS data is to average across the TE dimension prior to application of the standard 1D FFT (TE-averaging MRS; 8). The resulting TE-averaged ${ }^{1} \mathrm{H}$ MRS data (mathematically 
equivalent to the central row of a 2D J-resolved MRS dataset; 9) have a much simplified baseline structure due to the attenuation of $J$-coupled and macromolecule resonances. The improved resolution of glutamate (Glu; 8), glutamine (Gln; 10), and N-acetyl aspartylglutamate (NAAG; 11 ) has been documented using TE-averaging ${ }^{1} \mathrm{H}$ MRS at 3.0 Tesla.

Given the macromolecule signal attenuation, simplified spectral appearance and inherent metabolite $\mathrm{T}_{2}$ relaxation information, the objective of the present study was to develop a novel data processing scheme to estimate metabolite $\mathrm{T}_{2}$ relaxation times from TEaveraged ${ }^{1} \mathrm{H}$ MRS data. A specific focus was to develop a processing strategy using oversampled acquisition parameters that are finding increasing use for $2 \mathrm{D}{ }^{1} \mathrm{H}$ MRS measures $(6,12,13)$. The theory behind the procedures initially are introduced in the following section, and spectral simulations are employed to validate the proposed methods. The in vivo utility of the technique and its reproducibility are demonstrated using data obtained from the posterior-occipital cortex of healthy control subjects.

\subsection{Experimental}

\subsection{Theory}

TE-averaging across a range of incrementally spaced TE values results in an effective TE (i.e. the $\mathrm{TE}$ at the mean signal intensity during $\mathrm{T}_{2}$ decay) that is determined by a metabolite's proton $T_{2}$ relaxation time (8). The effective $T E$ for a metabolite $T_{2}$ relaxation time of $200 \mathrm{~ms}$ is around $219 \mathrm{~ms}$ when TE-averaging is performed over the 30 to $500 \mathrm{~ms}$ range using a TE increment ( $\Delta \mathrm{TE}$ ) of $10 \mathrm{~ms}$. For the same $\mathrm{T}_{2}$ value, the effective $\mathrm{TE}$ will be markedly different $(121 \mathrm{~ms})$ using the acquisition parameters that increasingly are being employed for oversampled 2D $J$-resolved ${ }^{1} \mathrm{H}$ MRS measurements (e.g. TE range $=31$ to 229 $\mathrm{ms}, \Delta \mathrm{TE}=2 \mathrm{~ms}$ ). It follows that averaging across selected TE ranges can produce metabolite signal attenuation factors that are predominantly governed by the proton $T_{2}$ relaxation time. This report proposes the use of TE-averaging procedures for estimating $\mathrm{T}_{2}$ relaxation times as follows. First, an array of incrementally spaced TE steps (NTE) are acquired as defined by equation (1)

$$
N T E=\left(\begin{array}{llll}
T E_{1} & T E_{2} & \cdots & T E_{n}
\end{array}\right),
$$

where $\mathrm{n}$ is the total number of individually obtained TE steps. Subsequently, a number of TE subarrays are extracted from the data. In principle, any number of subarrays can be drawn although the present study focuses on TE-averaging over two subarrays. Assuming monoexponential metabolite proton $\mathrm{T}_{2}$ relaxation time decay, the relative signal attenuation following TE-averaging across two subarrays can be expressed as shown in equation (2)

$$
\frac{1}{N 1} \sum_{i=k}^{n 1} \exp \left(\frac{-N T E(i)}{T_{2}}\right)=\frac{F}{N 2} \sum_{i=l}^{n 2} \exp \left(\frac{-N T E(i)}{T_{2}}\right),
$$

where $\mathrm{N} 1$ and $\mathrm{N} 2$ denote the lengths of the two subarrays, $k$ and $l$ are the selected initial TE steps, and $n l$ and $n 2$ are the final TE steps for the subarrays. Equation 2 introduces a 
correction factor, $F$, which is determined through TE-averaging over the two subarrays and subsequent calculation of the signal amplitude ratio. Once $F$ is calculated for a given metabolite proton resonance, equation (2) can be solved to yield an estimate of $T_{2}$ relaxation time.

\subsection{Simulation Procedures}

Point-resolved spectroscopy (PRESS; 14) ${ }^{1} \mathrm{H}$ MRS spectral simulations and subsequent data manipulations were performed using home-written scripts in MATLAB R2012a (The Mathworks, Natick, MA), in conjunction with the MATLAB Parallel Computing ${ }^{\mathrm{TM}}$ toolbox utilizing 4 dual-quad $2.8 \mathrm{GHz}$ Intel Xeon processors. The 3D localized simulations employed procedures described in previous reports $(10,15)$, with product-operator transformation matrices initially being calculated for each slice selection step using a $1 \%$ spatial resolution within the slice profile. The 3D simulations thus allowed for the incorporation of the experimental RF and gradient pulses, in addition to Zeeman, chemical shift and J-coupling effects for each metabolite. Spectra were calculated for a static magnetic field strength $\left(\mathrm{B}_{0}\right)$ of 2.89 Tesla $(\mathrm{T})$ corresponding to a proton magnetic resonance frequency of $\sim 123 \mathrm{MHz}$. The initial PRESS TE period (TE1) was fixed at $12 \mathrm{~ms}$ with the second TE period (TE2) incremented to realize the required total TE. The time-domain data were computed using 4096 complex points and a dwell time of $0.25 \mathrm{~ms}$.

A total of $100{ }^{1} \mathrm{H}$ MRS PRESS time-domain data were simulated for seventeen metabolites, including alanine (Ala), aspartate (Asp), Cre, $\gamma$-amino butyric acid (GABA), Gln, Glu, glycerophosphocholine (GPC), glycine (Gly), lactate (Lac), myo-inositol (Ins), and NAA, NAAG, phosphoryl choline (PCho), phosphoryl ethanolamine (PEtn), serine (Ser), scylloinositol (sIns), and taurine (Tau). Metabolite chemical shift values and $J$-coupling constants were taken from the literature (16). Due to coincident chemical shifts of creatine and phosphocreatine at 3.0 Tesla, methylene and methyl proton chemical shift data previously reported for phosphocreatine (16) were utilized for the simulation of Cre in the present study. Although separate spectral data were simulated for GPC and PCho, all $\mathrm{T}_{2}$ estimations were based on their composite $3.2 \mathrm{ppm}$ resonance, which hereafter is referred to as 'Cho' (total choline). PRESS data were generated with an initial TE (TE1+TE2) period of $31 \mathrm{~ms}$ $(\Delta \mathrm{TE}=2 \mathrm{~ms})$. All time-domain data were line broadened to a realistic in vivo linewidth (exponential filter; $6 \mathrm{~Hz}$ ) and subjected to signal weighting to reflect physiologic concentration ratios (16). For simulations, the line-broadening value of $6 \mathrm{~Hz}$ reflected the mean in vivo linewidth $(5.5 \pm 0.7 \mathrm{~Hz})$ measured for the parietal-occipital cortex $2.01 \mathrm{ppm}$ NAA methyl proton peak in spectra recorded from the human subjects introduced in section 2.4. Metabolite-specific $T_{2}$-relaxation time filters also were applied along the TE dimension using recent literature values (17). The same $\mathrm{T}_{2}$-relaxation time values were used for Glu, Gln, and GABA (181 ms), and for NAA and NAAG (258 ms), whereas a $T_{2}$ value of $200 \mathrm{~ms}$ was assumed for metabolites with no precedent literature values reported. Table 1 summarizes the metabolite concentration (amplitude weighting) and $\mathrm{T}_{2}$ values applied to the simulated data. Noise free composite time-domain data were produced for all 100 TE steps by summing the data for all fifteen metabolites on a TE-by-TE basis. 


\subsection{Evaluation of $\mathrm{T}_{2}$-Relaxation Time Calculation through TE-Averaging}

For the present study, two distinct subarrays were used to evaluate $\mathrm{T}_{2}$-relaxation time estimation through TE-averaging. The first and second subarrays used $k=1$ and $l=51$, respectively, with $n 1=50$, and $n 2=100$. The subarrays used $N 1=\mathrm{N} 2=50$ and were thus characterized by the TE ranges 31 to $129 \mathrm{~ms}(\Delta \mathrm{TE}=2 \mathrm{~ms})$ and 131 to $229 \mathrm{~ms}(\Delta \mathrm{TE}=2 \mathrm{~ms})$. A total of 1000 simulations were performed to evaluate the $T_{2}$ relaxation time estimation methods. Metabolite $\mathrm{T}_{2}$ relaxation times were pseudo-randomized to be $\pm 30 \%$ the values shown for Cre, Cho, and NAA in Table 1, with all other metabolite $\mathrm{T}_{2}$ relaxation times being the same between simulations. The simulations also allowed for the addition of complex random noise to each composite TE data step in order to match the in vivo situation. The amplitude of the added noise was determined from in vivo PRESS data (TE = $31 \mathrm{~ms}, 128$ signal averages) acquired from a voxel measuring $2.5 \times 2.5 \times 3.0 \mathrm{~cm}$ positioned in the posterior cingulate/occipital cortex of 10 healthy volunteers. The signal-to-noise ratio (SNR) from those measures was calculated in the frequency domain, and based on the maximum amplitude of the 2.01 ppm NAA resonance divided by the standard deviation of the noise level between 10 and $11 \mathrm{ppm}$ (mean in vivo SNR =243). Acquiring four signal averages per TE step, as typical for 2D J-resolved MRS in our laboratory, the SNR measured using the same approach would be approximately 43 . The maximum NAA amplitude from the first simulated time point $(\mathrm{TE}=31 \mathrm{~ms}$ ) was used to calculate the required noise standard deviation, permitting the computation and addition of noise arrays to each TE step. Unique noise arrays were generated for all 1000 simulations.

After TE-averaging across the subarrays, fast Fourier transformation (FFT) was applied and a Voigt line shape function was used to fit the $3.10 \mathrm{ppm}$ Cre, $3.21 \mathrm{ppm}$ Cho, and $2.0 \mathrm{ppm}$ NAA methyl peaks in the frequency domain (18). The nonlinear least-squares 'Isqnonlin' function provided with the MATLAB Optimization Toolbox ${ }^{\mathrm{TM}}$ was used to fit all frequency domain signals. Prior knowledge was provided in terms of initial parameter estimates, as well as lower and upper bounds for peak frequency, amplitude, linewidth, and Lorentzian/ Gaussian contribution. The fitted peak amplitudes calculated for both subarrays ultimately were used to calculate the correction factor, $F$. For the curve-based approach, the $\mathrm{T}_{2}$-curves were constructed using the logarithm of peak amplitudes outputted following spectral fitting in Linear Combination (LC) Model (19; version 6.2-2A). Each of the 100 TE steps were fitted using TE-specific simulated basis that were simulated using the methods outlined in section 2.2. Mild exponential apodization (line broadening $=1 \mathrm{~Hz}$ ) was applied to each TE step prior to LC Model spectral fitting. The linear fit of the logarithm of outputted signal amplitude for the multiple TE steps versus echo time allowed for the commonly employed estimation of metabolite $\mathrm{T}_{2}$ relaxation time (curve-based $\mathrm{T}_{2}$ estimation), which was compared directly with the TE-averaging approach.

\subsection{In vivo Data Acquisition}

All in vivo procedures were approved by the local Institutional Review Board, and met the criteria required for conducting studies in human subjects (see http://oshr.od.nih.gov, last accessed 06/20/2013). Ten healthy volunteers (mean age \pm standard deviation (SD) $=26 \pm 3$ years 6 females, 4 males) were recruited from the general public. MRI/MRS measurements were performed on two separate scan days for each subject, with scan 2 (S2) performed 
within one week of the baseline scan (S1). Data were acquired using a $2.89 \mathrm{~T}$ Siemens (Erlangen, Germany) TIM Trio ${ }^{\mathrm{TM}}$ whole-body MRI system, with a circularly polarized body coil used for RF transmission a (manufacturer-supplied) 12-channel phased array head coil used for signal reception. Data acquisition parameters were identical to those described elsewhere (13). Briefly, high-resolution $\mathrm{T}_{1}$-weighted, magnetization-prepared, rapid gradient echo (MP-RAGE TR/TE/TI = 2000/3.53/1100 ms; FOV = 256 x 256 × 224 mm isotropic $1 \mathrm{~mm}$ in-plane resolution) images were acquired to facilitate MRS voxel positioning within the parietal-occipital cortex (POC). The MRS voxel measured $25 \times 25 \times 30 \mathrm{~mm}^{3}$ for all subject data, obliqued along the sagittal plane and positioned bilaterally across the midline to cover predominantly gray matter. Voxel positioning at S2 was performed manually, by visually inspecting the corresponding voxel placement at $\mathrm{S} 1$ on axial and sagittal image slices reconstructed from baseline MP-RAGE dataset. TE-stepped ${ }^{1} \mathrm{H}$ MRS data were acquired from all subjects using the following acquisition parameters: PRESS sequence employing the same excitation and refocusing RF pulses as used for simulations, TE1 $=12$ $\mathrm{ms}, \mathrm{TR} / \mathrm{TE}=2400 / 31-229 \mathrm{~ms}, \Delta \mathrm{TE}=2 \mathrm{~ms}, 4$ signal averages per TE step, total acquisition time $=16$ minutes. Solvent water suppression was achieved using a three-pulse water elimination through $\mathrm{T}_{1}$-effects (WET; 20). Water unsuppressed data also were acquired with 2 signal averages collected for each TE step. The RF transmitter frequency was set to 3.0 and $4.7 \mathrm{ppm}$ for water suppressed and unsuppressed acquisitions, respectively.

\subsection{In vivo Data Processing and Analysis}

Brain extraction and tissue-type segmentation was applied to all MP-RAGE image data using the freely-available FMRIB software library (FSL; 21). MATLAB functions then were used to extract the MRS voxel and calculate within-voxel gray matter (GM), white matter (WM) and cerebrospinal fluid (CSF) tissue content for each subject. The within-voxel gray matter fraction was calculated as the ratio to total brain matter i.e. $100 \times \mathrm{GM} /(\mathrm{WM}+\mathrm{GM})$.

All ${ }^{1} \mathrm{H}$ MRS data were stored as individual channel data (Siemens TWIX files) and transferred to personal computer systems for spectral processing. Prior to channel recombination, eddy current distortions were accounted for in the time-domain using a reported time-domain technique (22), where the water suppressed data were corrected using channel-specific unsuppressed water data recorded at the same TE. Channel specific-signal weighting and recombination was performed as previously described (13). Residual water then was removed on a TE-by-TE basis using singular value decomposition (23). $\mathrm{T}_{2^{-}}$ relaxation times were estimated for Cre, NAA, and Cho using both TE-averaging principles and standard curve-based procedures. For the former approach, the subarrays employed were the same as those evaluated for the simulations, i.e. TE ranges 31 to $129 \mathrm{~ms}$ ( $\Delta \mathrm{TE}=2$ $\mathrm{ms}$ ), and 131 to $229 \mathrm{~ms}(\Delta \mathrm{TE}=2 \mathrm{~ms})$. For the second approach, the $\mathrm{T}_{2}$-curves were constructed using the logarithm of peak amplitudes outputted following spectral fitting LC Model as described for simulated spectra in section 2.3. All statistical analysis procedures were performed using MedCalc (version 12.5; Ostend, Belgium).

\subsection{Results}

Figure 1 generalizes the results from all 1000 simulations, overalying both the curve-based and TE-averaging $T_{2}$ relaxation time estimates versus the target metabolite $T_{2}$ values for the 
three metabolites. Figure 1 also presents the equations and corresponding $\mathrm{R}^{2}$ values resulting from linear curve fitting of each of the six plots. The mean target $\mathrm{T}_{2}$, curveestimated $\mathrm{T}_{2}$, and TE-averaging estimated $\mathrm{T}_{2}$ values for Cre were $148 \pm 25,147 \pm 25$, and $143 \pm 24 \mathrm{~ms}$, respectively. The corresponding values for Cho and NAA were $240 \pm 42,255$ \pm 47 , and $257 \pm 47 \mathrm{~ms}$, and $260 \pm 44,250 \pm 41,265 \pm 46 \mathrm{~ms}$, respectively. Paired t-tests showed that, for all three metabolites, statistical comparisons between target $T_{2}$ and estimated $\mathrm{T}_{2}$ were significantly different $(\mathrm{p}<0.01)$ for both approaches.

Figure 2(a) shows a pair of TE-averaged ${ }^{1} \mathrm{H}$ MRS datasets taken from the 1000 total simulations (see Figure 2 legend for more details). The two TE-averaged spectra shown were constructed using the two distinct subarrays described in section 2.3. Displayed in Figure 2(b) are the corresponding $\mathrm{T}_{2}$ semi-logarithmic curves and the calculated linear fits for Cho, Cre, and NAA. The mean GM fraction for the POC voxel was $68 \pm 4 \%$ (calculated using all 20 scans) with an inter-subject CV of $2 \%$. Figure 2(c) shows a pair of constructed TE-averaged ${ }^{1} \mathrm{H}$ MRS datasets recorded in vivo from the POC of a single subject. The qualitative similarity between those spectra and the simulated data in Figure 2(a) can be appreciated, particularly regarding the overall lineshape structures and SNR. The corresponding semi-logarithmic $\mathrm{T}_{2}$ curves and calculated linear fits for Cho, Cre, and NAA, also are presented in Figure 2(d). The mean $\mathrm{T}_{2}$ relaxation times for Cho, Cre, and NAA are summarized in Table 2.

Figure 3(a) shows a plot of the regression analysis of in vivo Cho, Cre, and NAA proton $\mathrm{T}_{2}$ relaxation times estimated using TE-averaging, versus the standard curve-based $\mathrm{T}_{2}$ estimation procedure $\left(\mathrm{R}^{2}=0.86\right)$. Figures $3(\mathrm{~b})-3(\mathrm{~d})$ display Bland-Altman plots for comparing method performance of TE-averaging and curve-based procedures for estimating Cho, Cre, and NAA $T_{2}$ values. Finally, the within-subject mean coefficients of variation (CV; calculated as the standard deviation $\div$ mean) for curve-based $\mathrm{T}_{2}$ estimates were 7,3 , and 3\%, for Cho, Cre, and NAA, respectively. The corresponding mean CV values calculated using TE-averaging for $\mathrm{T}_{2}$ estimates were 7,3 , and $4 \%$, for Cho, Cre, and NAA, respectively.

\subsection{Discussion}

The present proof-of-concept study demonstrates, for the first time, the use of TE-averaging procedures for estimating metabolite proton $\mathrm{T}_{2}$ relaxation times from datasets recorded with multiple echo times. The TE-averaging approach utilized two predefined TE subarrays, and the results were compared with the standard $\mathrm{T}_{2}$ curve-based fitting approach. Spectral simulations initially were used to demonstrate the validity of the TE-averaging technique, and the resulting data showed $\mathrm{T}_{2}$-estimation performance comparable with standard curvebased approaches for Cho, Cre, and NAA methyl protons at a range of randomized target $\mathrm{T}_{2}$ values $(\mathrm{N}=1000)$. This was evidenced by linear regression analysis and desirable slope $\left(>=0.95\right.$ ) and intercept $\left(<7 \%\right.$ of target $\mathrm{T}_{2}$ ) coefficients calculated for each metabolite using the TE-averaging method. The $\mathrm{T}_{2}$ estimation for the NAA methyl proton group was particularly improved using TE-averaging (slope, intercept $=1.03,-3.3$ ) when compared to curve-based estimation (slope, intercept $=0.93,8.9$ ). In general, both $T_{2}$ estimation methods conformed extremely well to linear fitting $\left(\mathrm{R}^{2}>=0.99\right)$. Random noise was included for all 
spectral simulations, using a noise level typically observed for $2 \mathrm{D} \mathrm{J}$-resolved ${ }^{1} \mathrm{H}$ MRS investigations within our laboratory (13).

Overall, the in vivo metabolite proton $\mathrm{T}_{2}$ values estimated using both TE-averaging and standard curve-based procedures showed a strong correlation $\left(\mathrm{R}^{2}=0.86\right)$, and also reflect precedent literature documenting Cho, Cre, and NAA $\mathrm{T}_{2}$ values at 3.0 Tesla $(17,24,25)$. Bland-Altman method comparisons infer that the two methods also can be used interchangeably for Cho, Cre, and NAA. However, the Cre and NAA $\mathrm{T}_{2}$ values estimated using standard curve-fitting method tended to be, on average, approximately $8 \%$ higher than the corresponding TE-averaging derived $\mathrm{T}_{2}$. As documented by Brief and coworkers (24), the sampling of a $T_{2}$ decay curve with a maximum $T E \approx T_{2}$ introduces a bias that overestimates metabolite $\mathrm{T}_{2}$. With this in mind, it is interesting to note that the Cre and NAA $T_{2}$ relaxation times estimated using TE-averaging are in excellent agreement with previously reported values. For example, the Cre $\mathrm{T}_{2}$ estimated using TE-averaging in the present study (154 ms) should be compared with the mean occipital cortical (predominantly gray matter) values reported by Brief et al. (159 ms, 24), Mlynarik et al. (152 ms, 25), and Ganji et al. (147 ms, 18). Likewise, the NAA $\mathrm{T}_{2}$ estimated using TE-averaging in the present study (256 ms) agrees well with the previously reported values ( $256 \mathrm{~ms}, \mathbf{2 4} ; 247 \mathrm{~ms}, \mathbf{2 5} ; 258$ $\mathrm{ms}, \mathbf{1 7}$ ). For Cho, the TE-averaging approach results in a comparable $\mathrm{T}_{2}$ estimation (within $3 \%$ ) when compared to curve-fitting procedures. The Cho $\mathrm{T}_{2}$ estimated using TE-averaging $(280 \mathrm{~ms})$ is also slightly higher than what has been previously documented using standard methods (249 ms, 24; $207 \mathrm{~ms}, \mathbf{2 5} ; 249 \mathrm{~ms}, \mathbf{1 7})$. However, a separate previous study reported higher Cho $\mathrm{T}_{2}$ relaxation time estimations $(265 \pm 29 \mathrm{~ms})$ in data recorded from MRS voxels containing higher gray matter content (26). Given the comparable performance of both methods for Cho $\mathrm{T}_{2}$ estimation in the present study simulations), the estimated in vivo $\mathrm{T}_{2}$ value may actually represent the present cohort examined, voxel dimensions and positioning, and within-voxel tissue composition. For all three metabolites, the $\mathrm{T}_{2}$ measurement reproducibility as measured by the within-subject $\mathrm{CV}$, were highly favorable $(<7 \%)$ and comparable between $\mathrm{T}_{2}$ measurement techniques.

There are several distinct advantages associated with using TE-averaging for estimating metabolite proton $T_{2}$ relaxation times. First, due to the attenuation of short $T_{2}$ macromolecule resonances and the additional macromolecule signal cancelation through the destructive interference of the phase-modulated ( $J$-coupled) resonances, TE-averaged ${ }^{1} \mathrm{H}$ MRS spectra are characterized by flatter baselines compared to single, short-moderate $\mathrm{TE}^{1} \mathrm{H}$ MRS data. A macromolecule $\mathrm{T}_{2}$ relaxation time of $40 \mathrm{~ms}$ (27) will result in effective TE values of 71 and $171 \mathrm{~ms}$ for the two subarrays used in the present study. Only $\sim 1$ to 17 $\%$ of the initial transverse magnetization remains following TE-averaging across the subarrays, and the minimal contribution from macromolecule resonances simplifies spectral fitting procedures. Conversely, discrete spectra recorded at different TE values are used to construct conventional $\mathrm{T}_{2}$ curves, and significant macromolecule peaks will be detectable at the shorter-moderate TE data points. Unless accounted for on a TE-by-TE basis, the macromolecule baseline are likely to affect spectral fitting of metabolite peak amplitude parameters, potentially degrading the precision of $\mathrm{T}_{2}$ calculation. A related limitation with respect to $\mathrm{T}_{2}$-curve analysis in the present study is that $\mathrm{LC}$ Model estimated the 
macromolecule contribution and baseline on a TE-by-TE basis. Second, the data quality in terms of metabolite peak SNR is significantly higher for TE-averaged datasets compared to the individual TE data elements, thus enhancing the precision of spectral peak fitting and metabolite $\mathrm{T}_{2}$ estimation. Third, the TE-averaging $\mathrm{T}_{2}$ calculation relies on the minimization of equation 2 as opposed to curve fitting methods. Once the correction factor $F$ has been calculated for a given metabolite, equation 2 can be rapidly solved (minimized) to estimate the $\mathrm{T}_{2}$ relaxation time without specialized fitting/minimization packages. Equation 2 infers a two-point metabolite proton $\mathrm{T}_{2}$ estimation procedure, although it should be noted that each data point (i.e. each subarray) contains amplitude information from a larger range of TE values with the simultaneous advantage of substantial macromolecule peak attenuation.

Future work should investigate the influence of the macromolecule resonances and baseline to metabolite $\mathrm{T}_{2}$ estimation using TE-averaging and standard curve based approaches. Metabolite-nulling techniques (28) could be used to generate TE-specific macromolecule basis functions, which then could be incorporated into the spectral fitting procedures for both TE-averaging and curve-based metabolite $\mathrm{T}_{2}$ estimation. Future work also should explore the effect of acquiring additional TE steps acquired at longer echo times to calculate metabolite $\mathrm{T}_{2}$ with conventional curve fitting, and to compare those results with the TEaveraging approach (24). TE-averaging ${ }^{1} \mathrm{H}$ MRS methods also have been reported for simplification and quantification of additional metabolite resonances including Glu (8) and NAAG (11), and we currently are optimizing similar post-processing approaches for calculating the $\mathrm{T}_{2}$ for these compounds. Note that for coupled spins such as the $\mathrm{C} 4$ methylene protons of Glu, additional information regarding $J$-coupling effects and its influence on the resulting spectral patterns in both subarrays is likely to be critical for calculating $\mathrm{T}_{2}$ relaxation time using TE-averaging approaches. The TE-averaging $\mathrm{T}_{2}$ estimation method conceivably could be applied to multi-voxel spectroscopic imaging data or standard proton MRI for whole-brain metabolite or water $\mathrm{T}_{2}$ mapping, respectively. The TE-averaging parameters in the present study (i.e. length, TE range, TE increment) were selected to ensure that the two subarrays showed zero overlap, thus limiting the inclusion of potentially contaminated data points in both subarray data. However, optimization of the subarray parameters for known metabolite $\mathrm{T}_{2}$ ranges also should be considered for future method development.

In conclusion, a novel method for metabolite proton $\mathrm{T}_{2}$ calculation at common clinical magnetic field strengths is presented, which is based on TE-averaging data acquisition and modified a processing pipeline. Advantages of this approach include built-in macromolecule resonance attenuation, in vivo $\mathrm{T}_{2}$ estimates closer to reported values when maximum $\mathrm{TE} \approx$ $\mathrm{T}_{2}$ (e.g. oversampled $2 \mathrm{D} \mathrm{J}$-resolved ${ }^{1} \mathrm{H}$ MRS), and the potential for $\mathrm{T}_{2}$ calculation of metabolite resonances otherwise inseparable in standard ${ }^{1} \mathrm{H}$ MRS spectra recorded in vivo.

\subsection{Disclosures}

Can be supplied at a later stage. 


\section{Abbreviations}

$\begin{array}{ll}\text { T2 } & \text { Spin-spin relaxation time constant } \\ \text { TE } & \text { echo time } \\ \text { PRESS } & \text { point-resolved spectroscopy } \\ \text { POC } & \text { posterior-occipital cortex } \\ \text { CSF } & \text { cerebrospinal fluid } \\ \text { GM } & \text { gray matter } \\ \text { WM } & \text { white matter } \\ \text { RF } & \text { radiofrequency } \\ \text { Ala } & \text { alanine } \\ \text { Asp } & \text { aspartate } \\ \text { Cho } & \text { choline-containing compounds } \\ \text { Cre } & \text { creatine } \\ \text { GABA } & \gamma \text {-amino butyric acid } \\ \text { Gln } & \text { glutamine } \\ \text { Glu } & \text { glutamate } \\ \text { Glx } & \text { glutamine plus glutamate } \\ \text { Ins } & \text { myo-inositol } \\ \text { Lac } & \text { lactate } \\ \text { MM } & \text { macromolecule } \\ \text { NAA } & \text { N-acetyl aspartate } \\ \text { NAAG } & \text { N-acetyl aspartyl glutamate } \\ \text { SI } & \text { scyllo-inositol } \\ \text { Tau } & \text { taurine } \\ \text { CV } & \text { coefficient of variation } \\ \end{array}$

\subsection{References}

1. Manton DJ, Lowry M, Blackband SJ, Horsman A. Determination of proton metabolite concentrations and relaxation parameters in normal human brain and intracranial tumours. NMR Biomed. 1995; 8(3):104-112. [PubMed: 8579997]

2. Ongur D, Prescot AP, Jensen JE, Rouse ED, Cohen BM, Renshaw PF, Olson DP. T2 relaxation time abnormalities in bipolar disorder and schizophrenia. Magn Reson Med. 2010; 63(1):1-8. [PubMed: 19918902]

3. Du F, Cooper A, Cohen BM, Renshaw PF, Ongur D. Water and metabolite transverse T2 relaxation time abnormalities in the white matter in schizophrenia. Schizophr Res. 2012; 137(1-3):241-245. [PubMed: 22356802] 
4. Tunc-Skarka N, Weber-Fahr W, Hoerst M, Meyer-Lindenberg A, Zink M, Ende G. MR spectroscopic evaluation of $\mathrm{N}$-acetylaspartate's $\mathrm{T} 2$ relaxation time and concentration corroborates white matter abnormalities in schizophrenia. NeuroImage. 2009; 48(3):525-531. [PubMed: 19573608]

5. Walker PM, Ben Salem D, Lalande A, Giroud M, Brunotte F. Time course of NAA T2 and ADC(w) in ischaemic stroke patients: 1H MRS imaging and diffusion-weighted MRI. J Neurol Sci. 2004; 220(1-2):23-28. [PubMed: 15140601]

6. Schulte RF, Boesiger P. ProFit: two-dimensional prior-knowledge fitting of J-resolved spectra. NMR Biomed. 2006; 19(2):255-263. [PubMed: 16541464]

7. Schulte RF, Lange T, Beck J, Meier D, Boesiger P. Improved two-dimensional J-resolved spectroscopy. NMR Biomed. 2006; 19(2):264-270. [PubMed: 16541465]

8. Hurd R, Sailasuta N, Srinivasan R, Vigneron DB, Pelletier D, Nelson SJ. Measurement of brain glutamate using TE-averaged PRESS at 3T. Magn Reson Med. 2004; 51(3):435-440. [PubMed: 15004781]

9. Bolan PJ, DelaBarre L, Baker EH, Merkle H, Everson LI, Yee D, Garwood M. Eliminating spurious lipid sidebands in 1H MRS of breast lesions. Magn Reson Med. 2002; 48(2):215-222. [PubMed: 12210929]

10. Prescot AP, Richards T, Dager SR, Choi C, Renshaw PF. Phase-adjusted echo time (PATE)averaging $1 \mathrm{H}$ MRS: application for improved glutamine quantification at $2.89 \mathrm{~T}$. NMR Biomed. 2012; 25(11):1245-1252. [PubMed: 22407923]

11. Zhang Y, Li S, Marenco S, Shen J. Quantitative measurement of N-acetyl-aspartyl-glutamate at 3 T using TE-averaged PRESS spectroscopy and regularized lineshape deconvolution. Magn Reson Med. 2011; 66(2):307-313. [PubMed: 21656565]

12. Northoff G, Walter M, Schulte RF, Beck J, Dydak U, Henning A, Boeker H, Grimm S, Boesiger P. GABA concentrations in the human anterior cingulate cortex predict negative BOLD responses in fMRI. Nat Neurosci. 2007; 10(12):1515-1517. [PubMed: 17982452]

13. Prescot AP, Renshaw PF. Two-dimensional J-resolved proton MR spectroscopy and prior knowledge fitting (ProFit) in the frontal and parietal lobes of healthy volunteers: assessment of metabolite discrimination and general reproducibility. J Magn Reson Imaging. 2013; 37(3):642651. [PubMed: 23055387]

14. Bottomley PA. Spatial localization in NMR spectroscopy in vivo. Ann N Y Acad Sci. 1987; 508:333-348. [PubMed: 3326459]

15. Choi C, Ganji SK, DeBerardinis RJ, Hatanpaa KJ, Rakheja D, Kovacs Z, Yang XL, Mashimo T, Raisanen JM, Marin-Valencia I, Pascual JM, Madden CJ, Mickey BE, Malloy CR, Bachoo RM, Maher EA. 2-hydroxyglutarate detection by magnetic resonance spectroscopy in IDH-mutated patients with gliomas. Nat Med. 2012; 18(4):624-629. [PubMed: 22281806]

16. Govindaraju V, Young K, Maudsley AA. Proton NMR chemical shifts and coupling constants for brain metabolites. NMR Biomed. 2000; 13(3):129-153. [PubMed: 10861994]

17. Ganji SK, Banerjee A, Patel AM, Zhao YD, Dimitrov IE, Browning JD, Brown ES, Maher EA, Choi C. T2 measurement of J-coupled metabolites in the human brain at 3T. NMR Boimed. 2012; 25(4):523-529.

18. Marshall I, Higinbotham J, Bruce S, Freise A. Use of Voigt lineshape for quantification of in vivo 1H spectra. Magn Reson Med. 1997; 37(5):651-657. [PubMed: 9126938]

19. Provencher SW. Estimation of metabolite concentrations from localized in vivo proton NMR spectra. Magn Reson Med. 1993; 30(6):672-679. [PubMed: 8139448]

20. Ogg RJ, Kingsley PB, Taylor JS. WET, a T1- and B1-insensitive water-suppression method for in vivo localized 1H NMR spectroscopy. J Magn Reson Ser B. 1994; 104(1):1-10. [PubMed: 8025810]

21. Smith SM, Jenkinson M, Woolrich MW, Beckmann CF, Behrens TE, Johansen-Berg H, Bannister PR, De Luca M, Drobnjak I, Flitney DE, Niazy RK, Saunders J, Vickers J, Zhang Y, De Stefano N, Brady JM, Matthews PM. Advances in functional and structural MR image analysis and implementation as FSL. NeuroImage. 2004; 23(Suppl 1):S208-219. [PubMed: 15501092]

22. Klose U. In vivo proton spectroscopy in presence of eddy currents. Magn Reson Med. 1990; 14(1): 26-30. [PubMed: 2161984] 
23. Cabanes E, Confort-Gouny S, Le Fur Y, Simond G, Cozzone PJ. Optimization of residual water signal removal by HLSVD on simulated short echo time proton MR spectra of the human brain. J Magn Reson. 2001; 150(2):116-125. [PubMed: 11384169]

24. Brief EE, Whittall KP, Li DK, MacKay AL. Proton T2 relaxation of cerebral metabolites of normal human brain over large TE range. NMR Biomed. 2005; 18(1):14-18. [PubMed: 15455460]

25. Mlynarik V, Gruber S, Moser E. Proton T (1) and T (2) relaxation times of human brain metabolites at 3 Tesla. NMR Biomed. 2001; 14(5):325-331. [PubMed: 11477653]

26. Traber F, Block W, Lamerichs R, Gieseke J, Schild HH. 1H metabolite relaxation times at 3.0 tesla: Measurements of T1 and T2 values in normal brain and determination of regional differences in transverse relaxation. J Magn Reson Imaging. 2004; 19:537-545. [PubMed: 15112302]

27. de Graaf RA, Brown PB, McIntyre S, Nixon TW, Behar KL, Rothman DL. High magnetic field water and metabolite proton T1 and T2 relaxation in rat brain in vivo. Magn Reson Med. 2006; 56(2):386-394. [PubMed: 16767752]

28. Kassem MN, Bartha R. Quantitative proton short-echo-time LASER spectroscopy of normal human white matter and hippocampus at 4 Tesla incorporating macromolecule subtraction. Magn Reson Med. 2003; 49(5):918-927. [PubMed: 12704775] 

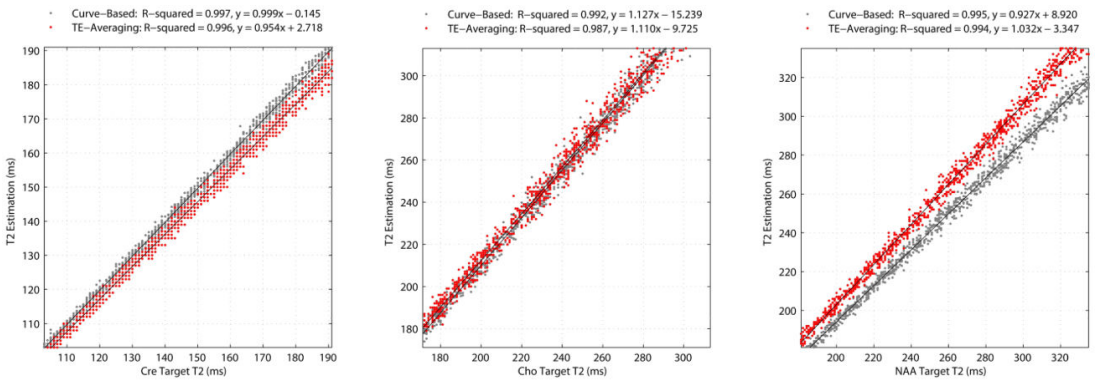

Figure 1.

Curve-based (gray data points) and TE-averaging (red data points) $\mathrm{T}_{2}$ relaxation time estimates versus the target Cre (left plot), Cho (middle plot), and NAA (right plot) $\mathrm{T}_{2}$ values for the total 1000 simulations. For each scatter plot and metabolite, the equations resulting from linear fitting are presented together with the coefficients of determination $\left(\mathrm{R}^{2}\right)$ at the top of each plot. 

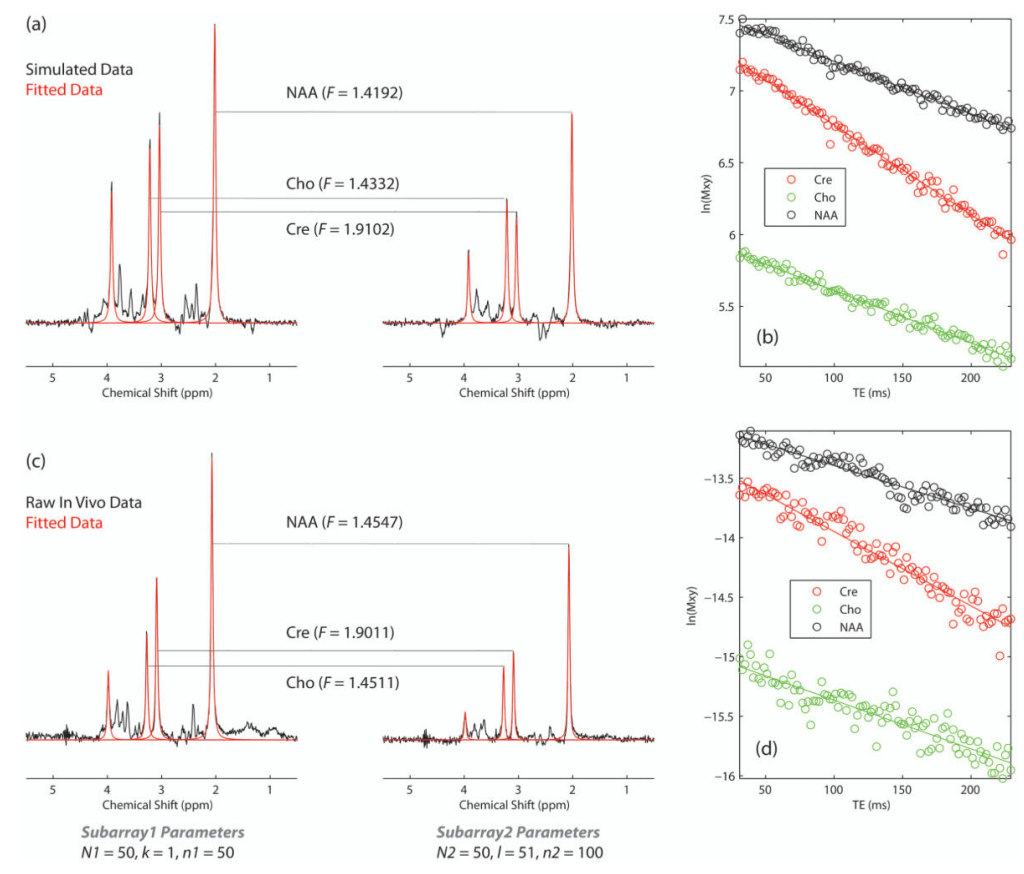

Figure 2.

(a) Simulated TE-averaged ${ }^{1} \mathrm{H}$ MRS data constructed from a single simulation using the parameters shown beneath each spectrum. The black line spectra are the raw spectral data and the Voigt fits for Cho, Cre, and NAA are overlaid (red line spectra). The horizontal lines illustrate the relative signal attenuation achieved following TE-averaging of the two subarrays, and the correction factors, $F$, subsequently were used in equation 2 for calculating Cho, Cre, and NAA methyl proton $\mathrm{T}_{2}$-relaxation times. (b) Standard semi-logarithmic plots used to estimate Cho, Cre, and NAA methyl proton $\mathrm{T}_{2}$-relaxation times (color-coded). All 100 TE steps were used and the estimated linear fits (solid lines) are shown for each metabolite. For this simulation step, the target $\mathrm{T}_{2}$ values for Cho, Cre, and NAA were 258, 159 , and $284 \mathrm{~ms}$, respectively. These metabolite $\mathrm{T}_{2}$ relaxation times were estimated to be 270,159 , and $288 \mathrm{~ms}$, and 274, 160, and $273 \mathrm{~ms}$ using TE-averaging and curve-based $\mathrm{T}_{2}$ estimation, respectively. (c) Representative TE-averaged ${ }^{1} \mathrm{H}$ MRS datasets from the POC of a single subject and constructed using the parameters shown beneath each spectrum. The black line spectra are the raw spectral data and the Voigt fits for Cho, Cre, and NAA are overlaid (red line specta). The horizontal lines illustrate the relative signal attenuation achieved following TE-averaging of the two subarrays, and the correction factors, $F$, subsequently were used in equation 2 for calculating in vivo Cho, Cre, and NAA methyl proton $\mathrm{T}_{2}$-relaxation times. (d) Standard semi-logarithmic plots used to estimate in vivo Cho, Cre, and NAA methyl proton $\mathrm{T}_{2}$-relaxation times (color-coded). All 100 TE steps were used and the estimated linear fits (solid lines) are shown for each metabolite. For this in vivo dataset, the curve-based approach estimated $\mathrm{T}_{2}$-relaxation times of 244,160 , and $278 \mathrm{~ms}$, for Cho, Cre, and NAA, respectively. The corresponding values estimated using TE-averaging procedures were 269, 156, and $267 \mathrm{~ms}$. 

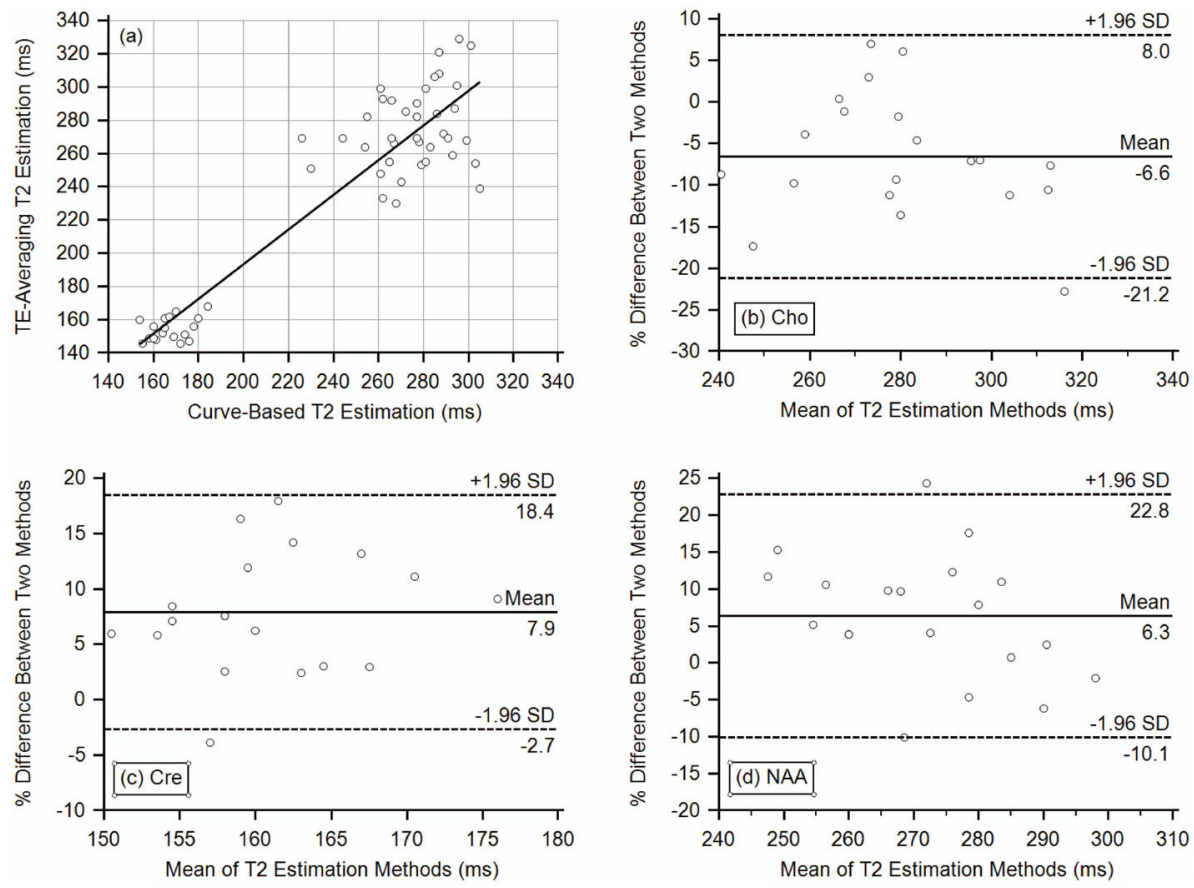

Figure 3.

(a) Regression analysis showing the TE-averaging $\mathrm{T}_{2}$ estimates versus curve-based $\mathrm{T}_{2}$ estimates for all twenty in vivo datasets. All Cho, Cre, and NAA data points were used to construct the data $\left(\mathrm{R}^{2}=0.86\right.$; slope $=1.04$; intercept $\left.=-15\right)$. (b) $-(\mathrm{d})$ Bland-Altman plots constructed for method comparison and evaluation of curve-based $\mathrm{T}_{2}$ estimates and TEaveraging $\mathrm{T}_{2}$ estimates for Cho, Cre, and NAA methyl protons. The vertical scale for each plot is shown as the $\%$ difference between the two $\mathrm{T}_{2}$ estimation methods, with the curvebased method designated as the standard (first) method. 


\section{Table 1}

Concentration (signal amplitude weighting) and $\mathrm{T}_{2}$-relaxation time parameters as applied to the simulated PRESS data for seventeen metabolites.

\begin{tabular}{ccc}
\hline Metabolite & Concentration $(\mathbf{m M})$ & $\mathbf{T}_{\mathbf{2}}$-relaxation time $(\mathbf{m s})$ \\
\hline Ala & 0.5 & 200 \\
Asp & 1 & 200 \\
Cre & 10 & $147^{*}$ \\
GABA & 1 & 181 \\
Gln & 4 & 181 \\
Glu & 10 & $181^{*}$ \\
Gly & 1 & 200 \\
GPC & 1.5 & 241 \\
Lac & 1 & 200 \\
Ins & 6 & $197^{*}$ \\
NAA & 12 & $258^{*}$ \\
NAAG & 1 & 258 \\
PCho & 1 & 241 \\
PEtn & 1 & 200 \\
Ser & 0.5 & 200 \\
sIns & 0.5 & 200 \\
Tau & 1 & 200 \\
\hline
\end{tabular}

denotes the use of metabolite $\mathrm{T}_{2}$-relaxation time values recently reported for $\mathrm{B}_{0}=3.0$ Tesla. 
Table 2

Group mean $\mathrm{T}_{2}$ proton relaxation time estimates for Cho, Cre, and NAA as measured using standard curvebased $\mathrm{T}_{2}$ estimation and TE-averaging principles.

\begin{tabular}{ccc}
\hline \multirow{2}{*}{ Metabolite } & \multicolumn{2}{c}{$\mathbf{T}_{\mathbf{2}}$ Estimation Method } \\
& Curve-Based (ms) & TE-Averaging (ms) \\
\hline Cho & $271 \pm 20$ & $280 \pm 20$ \\
Cre & $167 \pm 9$ & $154 \pm 7$ \\
NAA & $278 \pm 16$ & $256 \pm 19$ \\
\hline
\end{tabular}

